\title{
CONHECIMENTO SOBRE SEXUALIDADE EM IDOSAS FISICAMENTE ATIVAS
}

\author{
KNOWLEDGE ABOUT SEXUALITY IN PHYSICALLY ACTIVE OLDER WOMEN
}

\section{RESUMO}

O objetivo deste estudo foi analisar o conhecimento sobre sexualidade em idosas fisicamente ativas, assim como relacionar o mesmo com a prática de atividade física e o comportamento sedentário. Pesquisa transversal, na qual participaram 120 idosas praticantes de exercícios físicos em grupos sociais do município de Bom Sucesso/PR. Foi utilizado um questionário sociodemográfico, o Mini Exame do Estado Mental, a Escala de Atitudes e conhecimento sobre sexualidade no envelhecimento (ASKAS) e o International Physical Activity Questionnaire (IPAQ), versão curta. A análise dos dados foi realizada mediante os testes Kolmogorov-Smirnov. Kruskal-Wallis, "U” de Mann-Whitney e coeficiente de Spearman. Considerou-se um nível de significância de $\mathrm{p}<\mathrm{0,05}$. Verificou-se que as idosas com ensino médio completo possuem melhor conhecimento sobre sexualidade $(\mathrm{p}=\mathrm{0}, \mathrm{047})$ quando comparadas com as de menor nível de escolaridade. Que as idosas que ainda não são aposentadas tem melhor conhecimento sobre sexualidade $(\mathrm{p}=0,017)$ do que as aposentadas. Concluiu-se, assim, que o idoso com maior nível de escolaridade e que ainda possui situação ocupacional ativa apresentaram maior conhecimento sobre sexualidade. A atividade física parece não se relacionar com o conhecimento sobre a sexualidade de idosas.

Palavras-chave: Atividade motora. Envelhecimento. Gerontologia. Sexualidade.

\section{ABSTRACT}

The objective of this study was to analyze knowledge about sexuality in physically active older women, as well as to relate the same to the practice of physical activity and sedentary behavior. Cross-sectional research, in which 120 older women practicing physical exercises in social groups of Bom Sucesso, PR participated. A sociodemographic questionnaire, Mini Mental State Exam, Aging Sexual Knoledge and Attitudes Scale (ASKAS), and the International Physical Activity Questionnaire (IPAQ), short version, were used. Data analysis was performed using the KolmogorovSmirnov tests. Kruskal-Wallis, Mann-Whitney's "U” and Spearman's coefficient. A level of significance of $\mathrm{p}<0.05$ was considered. It was verified that older women with high school education have a better knowledge on sexuality $(\mathrm{p}=0.047)$ when

\footnotetext{
Daniel Vicentini de Oliveira

Universidade Estadual de Campinas (UNICAMP). E-mail: d.vicentini@hotmail.com Thais Gabriela Marques

Faculdade Metropolitana de Maringá (FAMMA). E-mail: thaisbsu@hotmail.com

Naelly Renata Saraiva Pivetta

Universidade Estadual de Campinas (UNICAMP).E-mail: naellypivetta@outlook.com

Débora Lee Vianna Paulo

Universidade Estadual de Campinas (UNICAMP). E-mail: deboraleevp@yahoo.com.br

José Roberto Andrade do Nascimento Júnior

Universidade Federal do Vale do São Francisco (UNIVASF). E-mail: jroberto.jrso1@gmail.com
} 
compared to those with a lower level of schooling. Older women who are not retired have better knowledge on sexuality $(\mathrm{p}=0.017)$ than retirees. It was concluded, thus, that the elderly with higher level of education and who still have an active occupational situation presented greater knowledge about sexuality. Physical activity does not seem to be related to the knowledge on the sexuality of the elderly.

Keywords: Motor activity. Aging. Gerontology. Sexuality.

\section{INTRODUÇÃO}

O envelhecimento é um fenômeno biológico, natural e dinâmico, caracterizado por um conjunto de alterações morfológicas, bioquímicas, psicológicas e fisiológicas que ocorrem de forma progressiva (Ferraresi et al. 2015). O declínio da função de órgãos vitais, modificação da aparência física e do erotismo gera dificuldade de adaptação aos novos papéis sociais, baixa-estima, desmotivação, autodepreciação e diminuição das relações afetivas (Fechine \& Trompieri 2015, Silva 2016). No entanto, os sentimentos e as sensações não sofrem decadência, o que torna possível ao idoso manter sua sexualidade, por exemplo, até o fim da sua vida, como afirma Ujvari et al. (2016). Embora o idoso enfrente déficits hormonais e alterações morfológicas no genital, a ciência afirma que não existem razões fisiológicas que impeçam a vida sexual ativa na terceira idade (Silva, 2016).

A sexualidade não se restringe à manipulação dos órgãos genitais e cada idoso expressa sua sexualidade com base em suas crenças, valores e cultura (Silva, 2016). A atividade sexual é um forte indicativo de qualidade de vida para o idoso, pois reflete o bem-estar subjetivo do indivíduo em relação a sua posição na vida, no contexto social em que vive e em relação às suas expectativas (Fleck, 200o; Viana \& Madruga, 2008).

Assim como a sexualidade, culturalmente, a atividade física parece, erroneamente, estar vinculada à juventude. No entanto, sua prática regular é fundamental para o bom funcionamento da mente e do organismo e implica diretamente sobre a qualidade de vida (Fernandes, 2014). Quando praticada regularmente, a atividade física pode promover a saúde do idoso, melhorando suas capacidades, percepções e vivências, sua sexualidade, independência e autonomia (Elias et al. 2012). E Estudos sugerem que idosos fisicamente ativos tendem a ser sexualmente mais ativos quando comparados à idosos sedentários (Cardoso et al. 2012; Vaz \& Nodin, 2012).

Considerando a importância de aprofundar o conhecimento sobre a temática relacionada ao envelhecimento, à sexualidade e à atividade física, o objetivo deste estudo foi analisar o conhecimento sobre sexualidade em idosas fisicamente ativas, assim como relacionar o mesmo com a prática de atividade física e o comportamento sedentário. 


\section{MÉTODOS}

Trata-se de uma pesquisa quantitativa, observacional, analítica, de cunho transversal.

\section{Participantes}

Participaram do estudo, 120 idosas, escolhidas de forma intencional e por conveniência, praticantes de exercícios físicos em grupos sociais do município de Bom Sucesso/PR. Foram incluídas idosas com idade igual ou superior a 60 anos, com capacidade de fala e audição preservadas, que permitiam a aplicação dos questionários.

Foi utilizado o Mini exame do estado mental (MEEM) para excluir idosas com déficits cognitivos relevantes. O MEEM é composto por questões agrupadas em sete categorias: orientação temporal, orientação espacial, registro de três palavras, atenção e cálculo, recordação das três palavras, linguagem e capacidade viso construtiva. As notas de corte utilizadas para exclusão pelo MEEM foram: 17 para as analfabetas; 22 para idosas com escolaridade entre 1 e 4 anos; 24 para as com escolaridade entre 5 e 8 anos e 26 os que tinham 9 anos ou mais anos de escolaridade. Estes pontos de corte foram baseados nos critérios de Brucki et al. (2003). Correspondem à média obtida por esses autores para cada faixa de escolaridade, menos um desvio padrão. Idosas classificadas abaixo do ponto de corte específico para sua escolaridade, foram excluídas. Foram excluídas, também, as idosas com problemas neurológicos que impossibilitaram a coleta de dados a partir dos instrumentos selecionados.

\section{Instrumentos}

O instrumento sociodemográfico foi constituído por questões referentes à idade (faixa etária: 6o a 69 anos; 70 a 79 anos; 80 anos ou mais), situação conjugal (com companheiro; sem companheiro), renda mensal em salário mínimo (SM) de referência do ano de 2017 ( $\mathrm{R} \$$ 937,0o) (1 a 2 SM; 2,1 a 3 SM; mais de 3 SM), escolaridade (analfabeta; ensino fundamental incompleto; ensino fundamental completo; ensino médio completo ou superior), raça (branca; negra; outra), aposentadoria (sim; não), percepção de saúde (boa; regular; ruim), tempo de prática de exercício físico (3 meses a 1 ano; 1,1 a 3 anos; mais de 3 anos).

Para avaliação do conhecimento sobre sexualidade na terceira idade, foi utilizada a Escala de Atitudes e conhecimento sobre sexualidade no envelhecimento (Aging Sexual Knowledge and Attitudes Scale - ASKAS). Foram utilizadas apenas as questões relacionadas ao conhecimento sobre sexualidade. Essa parte é composta de 20 questões, sendo que a pontuação baixa indica alto conhecimento sobre a sexualidade na velhice, cujas alternativas de respostas são: verdadeiro= 1 ponto, falso $=2$ pontos e não sei $=3$ pontos (Viana, et al. 2010)

O nível de atividade física das idosas foi avaliado utilizando-se a versão curta do Questionário Internacional de Atividade Física- (IPAQ). Este instrumento avalia 
atividades físicas realizadas no tempo de lazer, como deslocamento de um lugar ao outro, serviços domésticos e atividades ocupacionais. Foi considerado o tempo de 150 minutos semanais de atividades físicas para a idosa ser classificada como fisicamente ativa; para menos de dez minutos semanais, foi considerada sedentária e, aquelas que realizaram ao menos dez minutos, mas não alcançarem os 150 minutos, foram consideradas insuficientemente ativas. O comportamento sedentário foi avaliado por meio do tempo médio sentado em um dia de semana e em dia de final de semana (Matsudo et al. 2001).

\section{Coleta dos dados}

Primeiramente, foi feito contato com o (a) responsável por cada grupo social do município de Bom Sucesso/PR. Após a autorização de dois deles, a coleta de dados foi realizada em dias e horários diversos, de acordo com a disponibilidade dos grupos. As idosas foram abordadas antes e após a realização do programa de atividades e exercícios.

Os dados foram coletados na forma de entrevista por dois pesquisadores previamente treinados e após uma coleta piloto com 10 idosos. $\mathrm{O}$ fato de ser aplicado de forma oral explica a necessidade da exclusão de idosas que não tenham capacidade de audição, fala e compreensão preservadas. As idosas foram informadas quanto à justificativa, objetivos e procedimentos a serem realizados, conforme orientações para pesquisa com seres humanos constantes na Resolução 196/96 do Conselho Nacional de Saúde. As idosas que aceitaram participar da pesquisa assinaram o Termo de consentimento Livre e Esclarecido (TCLE).

Esta pesquisa foi aprovada pelo Comitê de ética em pesquisa do Centro Universitário de Maringá (UNICESUMAR) por meio do parecer número $1.769 .644 / 2016$.

\section{Análise dos dados}

A análise dos dados foi realizada por meio do Software SPSS 22.0. Ela foi realizada mediante uma abordagem de estatística descritiva e inferencial. Foram utilizadas frequência e percentual como medidas descritivas para as variáveis categóricas. Para as variáveis numéricas, inicialmente foi verificada a normalidade dos dados por meio do teste Kolmogorov-Smirnov. Como os dados não apresentaram distribuição normal, foram utilizadas Mediana (Md) e Quartis (Q1; Q3) para a caracterização dos resultados. Na comparação dos conhecimentos sobre sexualidade em função das variáveis sociodemográficas e de saúde, foi utilizado o teste de KruskalWallis ou o teste "U” de Mann-Whitney. A correlação entre o nível de atividade física, o comportamento sedentário e os conhecimentos sobre sexualidade foi verificada por meio do coeficiente de Spearman. Considerou-se um nível de significância de $\mathrm{p}$ $<0,05$. 


\section{RESULTADOS}

Das 120 idosas avaliadas, nota-se (Tabela 1) a prevalência de idosas com idade entre 60 e 69 anos (42,5\%), sem companheiros (50,8\%), com renda mensal de um a dois SM (50,0\%), da cor preta $(49,2,0 \%)$ e que não são aposentadas (90,0\%). Observou-se, também, que a maioria das idosas possui ensino médio completo ou ensino superior $(39,2,0 \%)$.

Tabela 1 - Perfil sócio demográfico e de saúde das idosas praticantes de exercícios físicos em grupos sociais. Bom Sucesso, Paraná, Brasil, 2017.

\begin{tabular}{lcc}
\hline VARIÁVEIS & $f$ & $\%$ \\
\hline Faixa Etária & & \\
60 a 69 anos & 51 & 42,5 \\
70 a 79 anos & 44 & 36,7 \\
80 anos ou mais & 25 & 20,8
\end{tabular}

Situação conjugal

Com companheiro

Sem companheiro

61

50,8

Renda mensal
1 a 2 SM

2,1 a 3 SM

Mais de 3 SM

Escolaridade

Analfabeta

Fundamental Incompleto

Fundamental Completo

Médio Completo/Superior
60

45

15

\section{4}

31

28

47

Cor

\section{Branca}

Preta

Outras

\section{Aposentadoria}

Sim

Não
$\%$

42,5

0,8

9,2
0,8

50,0

37,5

12,5

11,7

25,8

23,3

39,2

35,0

49,2

1915,8

$\begin{array}{cc}12 & 10,0 \\ 108 & 90,0\end{array}$

Percepção de saúde

Boa

$84 \quad 70,0$

Regular

30

25,0

Ruim
6 


\begin{tabular}{lcc}
\hline VARIÁVEIS & $f$ & $\%$ \\
\hline Tempo de prática de EF & & \\
3 meses a 1 ano & 41 & 34,2 \\
1,1 a z anos & 58 & 48,3 \\
Mais de 3 anos & 21 & 17,5 \\
Nível de atividade física & & \\
Ativo & 100 & 83,3 \\
Irregularmente ativo & 20 & 16,7 \\
\hline
\end{tabular}

Nota: SM=Salário Mínimo; EF=Exercício Físico.

Em relação ao perfil de saúde (Tabela 1), verificou-se que a maioria das idosas possui percepção de saúde boa (70,0\%), pratica exercícios entre 1,1 e 3 anos $(48,3 \%)$ e é ativa fisicamente $(83,3 \%)$. A Tabela 2 apresenta o nível de atividade física, comportamento sedentário e conhecimento sobre sexualidade das idosas.

Tabela 2 - Nível de atividade física, comportamento sedentário e conhecimento sobre sexualidade das idosas praticantes de exercícios físicos em grupos sociais. Bom Sucesso, Paraná, Brasil, 2017.

\begin{tabular}{lcc}
\hline VARIÁVEIS & Md & Q1-Q3 \\
\hline Conhecimento sobre sexualidade & 35,0 & $31,0-38,0$ \\
Tempo sentado durante a semana (min.) & 210,0 & $180,0-240,0$ \\
Tempo sentado durante fim de semana (min.) & 240,0 & $240,0-270,0$ \\
Dias de caminhada & 3,0 & $3,0-3,0$ \\
Min. de caminhada p/ dia & 90,0 & $78,8-120,0$ \\
Min. de caminhada p/ semana & 270,0 & $240,0-360,0$ \\
Dias de atividade moderada & 2,0 & $2,0-2,0$ \\
Min. de atividade moderada p/ dia & 60,0 & $60,0-90,0$ \\
Min. de atividade moderada p/ semana & 120,0 & $120,0-180,0$ \\
Dias de atividade vigorosa & 1,0 & $0,0-1,0$ \\
Min. de atividade vigorosa p/ dia & 30,0 & $0,0-40,0$ \\
Min. de atividade vigorosa p/ semana & 30,0 & $0,0-60,0$ \\
\hline
\end{tabular}

Nota: Min=minutos.

Conforme os achados da Tabela 2, nota-se que as idosas realizavam poucas atividades vigorosas e moderadas durante a semana. No entanto, nota-se que, em relação à caminhada, as idosas apresentaram nos dias de caminhada Mediana de 3,0; com Mediana de 90,o e 270,o minutos de caminhada por dia e semana, respectivamente. Em relação ao comportamento sedentário, as idosas apresentaram 
Mediana de 210,o e 240,o para o tempo sentado durante a semana e fim de semana, respectivamente. Ainda se observou que as idosas apresentaram Mediana de 35,o nos conhecimentos sobre a sexualidade.

A Tabela 3 apresenta a comparação do conhecimento sobre sexualidade das idosas em função das variáveis sociodemográficas e de saúde.

Tabela 3 - Comparação do conhecimento sobre sexualidade das idosas praticantes de exercícios físicos em grupos sociais em função das variáveis sociodemográficas e de saúde. Bom Sucesso, Paraná, Brasil, 2017.

\begin{tabular}{|c|c|c|c|}
\hline GRUPOS & & $\begin{array}{c}\text { Conhecimentos } \\
\text { sobre sexualidade } \\
\text { Md }\left(Q_{1} ; Q_{3}\right) \\
\end{array}$ & $\mathrm{P}$ \\
\hline Escolaridade $^{\mathrm{b}}$ & $\begin{array}{l}\text { Analfabeto } \\
\text { Fundamental Incompleto } \\
\text { Fundamental Completo } \\
\text { Médio Completo/Superior† } †\end{array}$ & $\begin{array}{c}35,5(34,0 ; 39,0) \\
35,0(31,0 ; 40,0) \\
36,0(31,5 ; 38,0) \\
33,0(30,0 ; 37,0)\end{array}$ & $\mathbf{0}, \mathbf{0 4 7}{ }^{*}$ \\
\hline Faixa Etáriab & $\begin{array}{c}60 \text { a } 69 \text { anos } \\
70 \text { a } 79 \text { anos } \\
\text { 8o anos ou mais }\end{array}$ & $\begin{array}{l}33,0(30,8 ; 36,0) \\
36,0(32,0 ; 38,0) \\
36,0(32,0 ; 38,0)\end{array}$ & 0,342 \\
\hline Estado civil $^{\mathrm{b}}$ & $\begin{array}{l}\text { Com Companheiro } \\
\text { Sem companheiro }\end{array}$ & $\begin{array}{l}34, \mathrm{o}(31,0 ; 38,0) \\
35,0(32,0 ; 38,0)\end{array}$ & 0,469 \\
\hline Renda Mensal $^{\mathrm{b}}$ & $\begin{array}{c}1 \text { a } 2 \text { SM } \\
\text { 2,1 a } 3 \text { SM } \\
\text { Mais de } 3 \text { SM }\end{array}$ & $\begin{array}{l}36,0(32,0 ; 38,0) \\
34,0(30,0 ; 38,0) \\
33,0(30,0 ; 38,0)\end{array}$ & 0,212 \\
\hline Aposentadoria $^{a}$ & $\begin{array}{l}\text { Sim } \\
\text { Não }\end{array}$ & $\begin{array}{l}38,0(35,5 ; 41,3) \\
34,0(31,0 ; 38,0)\end{array}$ & $\mathbf{0}, \mathbf{0 1 7}{ }^{*}$ \\
\hline Percepção de saúde ${ }^{b}$ & $\begin{array}{l}\text { Boa } \\
\text { Regular } \\
\text { Ruim }\end{array}$ & $\begin{array}{l}35,0(31,0 ; 38,0) \\
34,5(31,0 ; 37,3) \\
36,0(32,0 ; 41,3)\end{array}$ & 0,633 \\
\hline Tempo de prática & $\begin{array}{l}3 \text { meses a } 1 \text { ano } \\
1,1 \text { a } 3 \text { anos } \\
\text { Mais de } 3 \text { anos }\end{array}$ & $\begin{array}{l}36,0(31,0 ; 39,0) \\
34,0(32,0 ; 38,0) \\
33,0(29,0 ; 38,0)\end{array}$ & 0,386 \\
\hline $\begin{array}{c}\text { Nível de atividade } \\
\text { física }\end{array}$ & $\begin{array}{c}\text { Ativo } \\
\text { Irregularmente ativo }\end{array}$ & $\begin{array}{l}35,0(31,0 ; 38,0) \\
34,5(30,3 ; 38,8)\end{array}$ & 0,986 \\
\hline
\end{tabular}

*Diferença significativa - $\mathrm{p}<0,05$ (Teste "U” de Mann-Whitney ${ }^{\mathrm{a}}$ e Kruskal-Wallis ${ }^{b}$ : Médio Completo/Superior com Analfabeto, Fundamental Incompleto e Fundamental Completo. SM: salário (s) mínimo (s).

Verificou-se diferença significativa nos conhecimentos sobre a sexualidade apenas para a escolaridade $(\mathrm{p}=\mathrm{o}, 047)$ e aposentadoria $(\mathrm{p}=\mathrm{o}, \mathrm{017})$. Estes resultados indicam que as idosas com ensino médio completo possuem melhor conhecimento sobre sexualidade quando comparadas com as idosas de menor nível de escolaridade 
e que as idosas que ainda não são aposentadas apresentam melhor conhecimento sobre sexualidade do que as aposentadas.

A Tabela 4 apresenta a correlação entre o nível de atividade física, o comportamento sedentário e o conhecimento sobre sexualidade.

Tabela 4 - Correlação entre o nível de atividade física, o comportamento sedentário e o conhecimento sobre sexualidade das idosas praticantes de exercício físico. Bom Sucesso,

Paraná, Brasil, 2017

\begin{tabular}{|c|c|c|c|c|c|c|c|c|c|c|c|c|}
\hline \multirow{2}{*}{ Variáveis } & \multicolumn{9}{|c|}{ Nível de atividade física } & \multicolumn{2}{|c|}{$\begin{array}{c}\text { Comportamento } \\
\text { sedentário }\end{array}$} & Sexualidade \\
\hline & 1 & 2 & 3 & 4 & 5 & 6 & 7 & 8 & 9 & 10 & 11 & 12 \\
\hline 1. & & 0,03 & $0,43^{*}$ & $0,21^{*}$ & $0,28^{*}$ & $0,26^{*}$ & 0,18 & 0,14 & 0,13 & $-0,25^{*}$ & $-0,16$ & $-0,03$ \\
\hline 2. & & & $0,81^{*}$ & $0,22^{*}$ & $0,52^{*}$ & $0,50^{*}$ & $0,43^{*}$ & $0,47^{*}$ & $0,38^{*}$ & $-0,23^{*}$ & $-0,17$ & 0,09 \\
\hline 3. & & & & $0,21^{*}$ & $0,55^{*}$ & $0,49^{*}$ & $0,35^{*}$ & $0,41^{*}$ & $0,32^{*}$ & $-0,27^{*}$ & $-0,23^{*}$ & 0,04 \\
\hline 4. & & & & & $0,34^{*}$ & $0,67^{*}$ & $0,46^{*}$ & $0,39^{*}$ & $0,38^{*}$ & $-0,36^{*}$ & $-0,31^{*}$ & $-0,07$ \\
\hline 5. & & & & & & $0,89^{*}$ & $0,37^{*}$ & $0,36^{*}$ & $0,35^{*}$ & $-0,34^{*}$ & $-0,28^{*}$ & $-0,01$ \\
\hline 6. & & & & & & & $0,50^{*}$ & $0,46^{*}$ & $0,45^{*}$ & $-0,41^{*}$ & $-0,38^{*}$ & $-0,03$ \\
\hline 7. & & & & & & & & $0,79^{*}$ & $0,81^{*}$ & $-0,55^{*}$ & $-0,42^{*}$ & 0,08 \\
\hline 8. & & & & & & & & & $0,91^{*}$ & $-0,53^{*}$ & $-0,48^{*}$ & 0,07 \\
\hline 9. & & & & & & & & & & $-0,53^{*}$ & $-0,45^{*}$ & 0,11 \\
\hline 10. & & & & & & & & & & & $0,63^{*}$ & $-0,02$ \\
\hline 11. & & & & & & & & & & & & 0,01 \\
\hline 12. & & & & & & & & & & & & \\
\hline
\end{tabular}

*Correlação Significativa - p < 0,05. Nota: 1. Dias de caminhada; 2. Min. caminhada p/ dia; 3. Min. caminhada p/ sem.; 4. Dias atv moderada; 5. Min. Ativ. Mod. p/ dia; 6. Min. Ativ. Mod. p/ sem; 7. Dias de atv. Vigorosa; 8. Min. Atv. vigorosa p/ dia; 9. Min. Atv. Vig. p/ sem.; 10. Tempo sentado durante a semana; 11. Tempo sentado durante fim de semana; 12.

Sexualidade.

Atv.: atividade(s).

Verificou-se (Tabela 4) que as variáveis de comportamento sedentário (tempo sentado durante a semana e fim de semana) apresentaram correlação significativa $(\mathrm{p}<\mathrm{0,05})$ e inversa com as variáveis relacionadas ao nível de atividade física. Tal resultado parece indicar que a realização de atividades físicas leves, moderadas e, principalmente, vigorosas, está relacionada com a redução do comportamento sedentário. $\mathrm{O}$ conhecimento sobre a sexualidade não apresentou correlação significativa ( $\mathrm{p}>0,05)$ com nenhuma das variáveis.

\section{DISCUSSÃO}

A presente pesquisa teve como objetivo analisar o conhecimento de idosas fisicamente ativas sobre sexualidade, assim como relacionar o mesmo com a prática de atividade física e o comportamento sedentário. Os resultados indicaram que maior nível de escolaridade e não estar aposentadas refletem em maior conhecimento 
sobre sexualidade. Ainda, o comportamento sedentário apresentou relação negativa com o nível de atividade física, verificando que quanto maior o comportamento sedentário na rotina da idosa, menor é o seu nível de atividade física. A sexualidade não apresentou relação com o nível de atividade física e o comportamento sedentário.

A Tabela 3 apresentou que as idosas com maior nível de escolaridade e que não recebem aposentadoria apresentam maior conhecimento sobre sexualidade quando comparadas com seus pares. Rheaume \& Mitty (2008) relatam que os adultos mais velhos não possuem informações precisas sobre sexualidade, uma vez que a educação sexual não estava no currículo de formação padrão e nem universitária dos adultos mais velhos de hoje. Os valores sexuais foram moldados por circunstâncias - por exemplo, economia, guerra, enculturação - e influenciados por mitos societários por exemplo, que a menopausa significa uma desaceleração do desejo sexual e perda de senso de feminilidade, que a atividade sexual deve ser iniciada pelo homem, existe apenas uma posição correta para relações sexuais - (Rheaume \& Mitty, 2008). O conhecimento limitado sobre o sexo e as atitudes sobre a sexualidade entre os adultos mais velhos estão intrinsecamente ligados (Rheaume \& Mitty, 2008). Os resultados do nosso estudo indicaram que as idosas com ensino médio completo e não aposentadas possuem melhor conhecimento sobre sexualidade quando comparadas com as idosas de menor nível de escolaridade e aposentadas. Isto remete que, mesmo com a ausência de educação sexual nas escolas, as idosas mais escolarizadas possuem mais condições de obter informações sobre o assunto, se tornando característica semelhante às idosas mais novas que não se aposentaram.

Uma manifestação dessa falta de conhecimento ou disposição para discutir assuntos sexuais é o aumento das taxas de diagnóstico de HIV/AIDS em adultos mais velhos. Nos adultos mais velhos, o diagnóstico de HIV/AIDS tende a ser feito mais tarde, o curso da doença é mais rápido e o prognóstico é mais fraco. Melhorar a educação sobre HIV/AIDS para adultos mais velhos pode ser uma estratégia eficaz para reduzir essas infecções (Rheaume \& Mitty, 2008), uma vez que esta população apresenta divergências sobre o conhecimento do assunto (Nascimento et al. 2013).

Em uma revisão integrativa, Alencar et al. (2014) evidenciaram que além das modificações fisiológicas que o corpo apresenta com o decorrer dos anos e que podem interferir na prática sexual, a cultura da assexualidade e o preconceito social com os mais velhos favorecem a construção do estereótipo de que a sexualidade está designada aos mais jovens, repreendendo, em idosos, desejos e vontades no campo sexual. Assim, os autores apontam a educação em saúde como uma estratégia na construção de conceitos que visualizem o idoso como indivíduo livre para vivenciar sua sexualidade desprendida de mitos e preconceitos que se solidificaram socialmente. Estas ações educativas devem envolver idosos e não idosos, pois o envelhecimento é inerente ao ser humano; questões sobre a sexualidade precisam ser discutidas no percurso de todas as etapas da vida. Nesta perspectiva, a atividade física durante a terceira idade é outro assunto a ser discutido com todos.

O presente estudo apresentou que o nível de atividade física no grupo da terceira idade possui relação negativa com o comportamento sedentário, em que quanto 
mais presente é o comportamento sedentário, menor é o nível de atividade física da idosa (Tabela 4), não apresentando relação com o conhecimento sobre sexualidade. A procura por atividades físicas na terceira idade é algo necessário para a boa saúde física do idoso, possibilitando melhoras fisiológicas e psicológicas, resultando em uma qualidade de vida mais satisfatória (Cordeiro et al. 2014). Entretanto, a falta de interesse na prática da atividade física é muito relatada entre os idosos, podendo ser um fator que leve ao aumento do comportamento sedentário (Carvalho et al. 2017).

Estudo de Cordeiro et al. (2014) apontou que idosos mais ativos apresentam maiores níveis de atividade física, maior autonomia em suas atividades de vida diária e melhor capacidade funcional quando comparados a idosos sedentários e insuficientemente ativos. Isto vai ao encontro do presente estudo, em que foi verificado que quanto maior o comportamento sedentário da idosa, menor é o seu nível de atividade física. Assim, a promoção da prática de atividade física voltada a terceira idade deve ser incentivada pela sociedade de uma maneira geral, para que, assim, o idoso possa chegar nesta fase da vida sabendo de todas as vantagem desta prática e assimilar a melhora de sua própria saúde.

Apesar das contribuições deste trabalho, limitações necessitam ser destacadas. Primeiro, a amostra ter sido realizadas apenas com idosas mulheres, não compreendendo como ocorre o conhecimento da sexualidade, o nível de atividade física e o comportamento sedentário de idosos homens. Segundo, ainda a respeito da amostra, por terem sido selecionadas apenas idosas participantes de grupos de exercícios físicos. Assim, sugere-se a investigação de idosos não ativos, por apresentarem características e comportamentos específicos relacionados as variáveis investigadas.

\section{CONCLUSÃO}

Concluiu-se que, a idosa com maior nível de escolaridade e que ainda possui situação ocupacional ativa apresentou maior conhecimento sobre sexualidade. Além disso, percebeu-se que a realização de atividades físicas leves, moderadas e principalmente vigorosas pode ser considerada um fator interveniente na redução do comportamento sedentário de idosos fisicamente ativos. A atividade física parece não se relacionar com o conhecimento sobre a sexualidade de idosas.

\section{REFERÊNCIAS}

ALENCAR, Danielle. Ana Paula de Oliveira Marques. Márcia Carréra Campos Leal Júlia de Cássia Miguel Vieira. (2014). "Fatores que interferem na sexualidade de idosos: uma revisão integrativa.” Ciência E Saúde Coletiva 19(8):3533-3542. 
BRUCKI, Sonia MD Nitrini. Ricardo Caramelli. Paulo Bertolucci. Paulo Henrique Ferreira Okamoto. Ivan H. (2003). Sugestões para o uso do mini-exame do estado mental no Brasil." Arquivos de Neuro-psiquiatria. 61(3B): 777-781.

CARDOSO, Fernando Luiz. Mazo, Giovana Zarpellon. Silveira, Rozana Aparecida da. Virtuoso, Janeisa Franck. Menezes, Enaiane Cristina. (2012). "Da juventude à velhice: sexualidade de idosos praticantes de atividade física." Arq catarin med [Internet] 41(1): 34-40.

CORDEIRO, Juliana Del Castillo. Bruna Lencina Freitas. Caroline Silva de Gonçalves. Marisa Pereira. (2014). "Effects of physical activity in declarative memory, functional capacity and quality of life in elderly." Revista Brasileira de Geriatria e Gerontologia 17(3): 541-552.

CARVALHO, Dilma Aurélia Brito. Aline Freitas Dos Santos. Marcos Antônio Pereira de Siqueira Nogueira. Fabiana Ranielle de Moura Sá. Guilherme Guarino de Oliveira Neto. Joaquim Guerra de Carvalho. Maria do Carmo dos Santos. Enivaldo Pereira. (2017). "Prevalência da prática de exercícios físicos em idosos e sua relação com as dificuldades e a falta de aconselhamento profissional específico." Revista Brasileira de Ciência e Movimento 25(1).

ELIAS, Rui Gonçalves Marques et al. (2012). "Aptidão física funcional de idosos praticantes de hidroginástica". Revista Brasileira de Geriatria e Gerontologia., 15(1): 79-86.

FECHINE \& TROMPIERI, Basílio Rommel Almeida \& Nicolino. (2015). "O processo de envelhecimento: as principais alterações que acontecem com o idoso com o passar dos anos." InterSciencePlace 1(20).

FERNANDES, Barbara Lourenço Vargas. (2014). "Atividade Física no processo de envelhecimento." Revista Portal de Divulgação (40).

FERRARESI, Juliana Rizzatto. Melina Galetti Prata. Marcos Eduardo Scheicher. (2015). "Avaliação do equilíbrio e do nível de independência funcional de idosos da comunidade." Rev. bras. geriatr. gerontol 18(3): 499-506.

FLECK, Marcelo Pio de Almeida. (200o). "The World Health Organization instrument to evaluate quality of life (WHOQOL-10o): characteristics and perspectives." Ciência E Saúde Coletiva 5(1): 33-38.

FOLSTEIN, Marshal F. Susan E Folstein. Paul R McHugh. (1975). ““Mini-mental state”: a practical method for grading the cognitive state of patients for the clinician.” Journal of psychiatric research 12(3): 189-198. 
MATSUDO, Sandra, Araújo T, Matsudo V, Andrade D, Andrade E, Oliveira LC, et al. "Questionário Internacional De Atividade Física (Ipaq): Estupo De Validade E Reprodutibilidade No Brasil. " (2001). Revista Brasileira de Atividade Física E Saúde 6(2):5-18.

MONTEIRO, Thuane Jaloto. Larissa Alexsandra da Silva Neto Trajano. Danieli Silva Carvalho Lauriane de Assis Proença Pinto Eduardo Tavares Lima Trajano. (2016). "Avaliação do conhecimento sobre HIV/AIDS em grupo de idosos através do QHIV3I." Geriatrics, Gerontology and Aging 10(1): 29-33.

NASCIMENTO, Rodolfo Gomes. Evanildo Lopes Monteiro. Laiana Soeiro Ferreira. Zeneide Lima Santos. (2013). "Nível de conhecimento de idosos comunitários em relação ao HIV/Aids: estudo exploratório na rede básica de saúde de Belém, Pará, Brasil." Revista Brasileira de Ciências do Envelhecimento Humano 1o(1).

RHEAUME \& MITTY, Chris \& Ethel. (2008). "Sexuality and intimacy in older adults." Geriatric Nursing 29(5): 342-349.

SILVA, Renata Maria Ortiz. (2016). "A sexualidade no envelhecer: um estudo com idosos em reabilitação.” Acta fisiátrica 10(3): 107-112.

UJVARI, Amanda Bufalo. Márcia Regina Benedeti. Daniel Vicentini de Oliveira. Ana Paula Serra de Araújo. (2016). "Quociente sexual feminino (QSF)- avaliando a sexualidade de idosas do município de Ivaiporã." Revista Thêma et Scientia 5(1): 99104 .

VAZ \& NODIN, Raquel Almeida \& Nuno. (2012). "A importância do exercício físico nos anos maduros da sexualidade." Analise psicológica. 23(3):329-339.

VIANA, Helena Brandão. Edinêis de Brito Guirardello. Vera Aparecida Madruga. (2010). Traduação e adaptação cultural da Escala Askas - Aging Sexual Knoledge and Attitudes Scale em idosos brasileiros. 19(2): 238-215

VIANA \& MADRUGA, Helena Brandão \& Vera Aparecida. (2008). "Sexualidade, qualidade de vida e atividade física no envelhecimento." Conexões.

Recebido: 13.12.2017

Aceito: 10.10 .2018 